\title{
(2) OPEN ACCESS \\ What matters to people with multiple long-term conditions and their carers?
}

\author{
Gemma Spiers (1) , ${ }^{1}$ Elisabeth Boulton, ${ }^{2}$ Lynne Corner, ${ }^{1}$ Dawn Craig, ${ }^{1}$ Stuart Parker, ${ }_{1}^{1}$ \\ Chris Todd, ${ }^{2}$ Barbara Hanratty ${ }^{1}$
}

${ }^{1}$ Faculty of Medical Sciences, Newcastle University, Newcastle upon Tyne, UK

${ }^{2}$ Faculty of Biology, Medicine and Health, The University of Manchester, Manchester, UK

\section{Correspondence to}

Dr Gemma Spiers, Newcastle University, Newcastle upon Tyne, Tyne and Wear, UK; gemma-frances.spiers@ newcastle.ac.uk

GS and EB are joint first authors.

Received 12 July 2021

Accepted 1 December 2021

\begin{abstract}
Background The number of people living with multiple long-term conditions is increasing worldwide. This presents challenges for health and care systems, which must adapt to meet the needs of this population. This study drew on existing data to understand what matters to people living with multiple long-term conditions and identify priorities for future research.
\end{abstract}

Methods Two studies were conducted. (1) A secondary thematic analysis of interview, survey and workshop data collected from the 2017 James Lind Alliance Priority Setting Partnership for Older People with Multiple Conditions, and patient and public involvement workshops; (2) a review of ongoing research and published research priorities, relating to older people $(80+)$ living with multiple long-term conditions.

Findings Older people with multiple long-term conditions identified a number of key concerns: access to care, support for both the patient and their carer, physical and mental health and well-being and identifying opportunities for early prevention. The review identified no published research priorities or ongoing research focusing specifically on populations aged over 80 years with multiple long-term conditions.

Conclusion Older people living with multiple long-term conditions experience care that is inadequate for their needs. A holistic approach to care that extends beyond treating single conditions will ensure wide-ranging needs are met. As multimorbidity rises worldwide, this is a critical message for practitioners across health and care settings. We also recommend key areas that should be given greater focus in future research and policy to inform effective and meaningful forms of support for people living with multiple long-term conditions.

\section{INTRODUCTION}

One-third of the global population are estimated to be living with two or more long-term health conditions, commonly termed multimorbidity. ${ }^{1-3}$ In the UK, multimorbidity has been described as the greatest clinical challenge facing the National Health Service and social care sector. The prevalence of multimorbidity rises with age, is more common among women, ${ }^{4}$ and in people of lower socioeconomic status. ${ }^{5} 6$ People with multimorbidity are more likely to have a poor quality of life, spend time in hospital ${ }^{8}$ and have an increased risk of death. ${ }^{9}$

The rising number of people living with multiple long-term conditions (MLTCs) presents challenges to current healthcare systems that are designed to treat and manage single health conditions. ${ }^{10}$
Patient-centred approaches may be hindered by the silo working associated with medical specialties. ${ }^{11}$ People living with MLTCs often report difficulties accessing timely, coordinated care. ${ }^{12}{ }^{13}$ Multiple appointments with different services increase the burden of treatment, ${ }^{14}$ compromising the overall quality of care that people receive. Practitioners face the challenge of coordinating support across primary, community, secondary and tertiary care settings. ${ }^{1115}$ Across health and social care, there is increasing recognition of the need to move away from a single disease-based model of care to optimise support for those with MLTCs. ${ }^{10}{ }^{16}$ Integration of health and social care services may deliver effective support, but implementation can be hampered by disease-specific approaches in medicine. ${ }^{17}$ As populations age, health and social care services will need to find ways to respond to the growing number of people living with MLTCs, to contain costs and demand. ${ }^{18-20}$

In 2017, the James Lind Alliance (JLA) Priority Setting Partnership (PSP) worked with older people, carers and a range of health and social care professionals to identify a set of priorities for future research on interventions for people with MLTCs (see box 1). ${ }^{21}$ This involved an initial information gathering exercise, where identified concerns and questions were cross-checked with existing evidence to identify a 'long-list' of 97 potential research questions. In consultation with stakeholders, these questions were reduced to a shortlist of 21 questions, and the top 10 ranked in order of priority. Most urgent was a need for research to identify ways to improve health and social care support for people with MLTCs. Understanding the perspectives and experiences of people living with MLTCs is critical to improve the care and support for this population. ${ }^{22}$ Others have also advocated for greater focus on individual experience, rather than on multimorbidity as a biomedical concept. ${ }^{23}$ At present, however, there is a substantial gap in our understanding of how multimorbidity impacts on the people who live with it, and their carers. ${ }^{24}$ In response to this gap, we used the data that were collected to support the JLA PSP exercise, to understand the experiences of older people living with MLTCs. The purpose of this study was to highlight the key issues and areas that are most important to this population to ensure these are considered in future research. This paper reports the findings of this work.

\section{METHODS}

We drew on the following data for the findings presented here: (1) qualitative data collected 
Box 1 Priorities identified by JLA PSP multiple conditions in later life

\section{JLA PSP on multiple conditions in later life, top 10 priorities}

Professional, public, care recipient and carer views Optimising health, social care and voluntary sectors to meet needs.

Effective and cost effective ways to reduce social isolation.

Supporting carers to maintain physical and psychological wellbeing.

Effective, cost effective and acceptable to improve

psychological well-being.

Enabling independent living effectively and acceptably.

Effective, cost effective and acceptable strategies for

preventing multiple conditions.

Effective, cost effective and acceptable form of exercise

therapy. How does this affect outcomes?

Recognising and managing frailty. Would this lead to

improved quality of life?

Optimising delivery of Comprehensive Geriatric Assessment.

Perception and management of falls risk. Addressing fear of falling effectively.

through the 2017 JLA PSP and from patient and public involvement (PPI) workshops conducted in 2019; and (2) data from a scoping review of existing research priorities for this population.

\section{Qualitative data from the JLA PSP and PPI workshops Participants}

Participants were people aged $80+$ yearsand their carers involved in the 2017 JLA PSP exercise on Multiple Conditions in Later Life. ${ }^{21}$ Survey participants were recruited via social media, charities and networks; interview participants were recruited via community services and care homes in the North East of England. The wide-ranging recruitment platforms, along with the use of interviews and paper surveys to facilitate participation for those without access to digital platforms, maximised involvement of older people who may otherwise be overlooked. Members participating in the VOICE PPI workshops were people were living with MLTCs and their carers, and aged between 45 and 89 years.

\section{Data collection}

Data from the 2017 JLA PSP were collected through (a) free-text responses to a survey conducted either online or by post; and (b) interviews with older people and carers. ${ }^{21}$ Additional data were collected in 2019 through two workshops with older people and carers to explore experiences of living with MLTCs and the challenges posed by the care and treatment of those conditions. The workshops were facilitated through VOICE, a network of citizens, patients, carers and members of the public, who contribute their lived experiences to health and care research on unmet needs and priorities (https://www.voice-global.org/).

\section{Data analysis}

The data obtained through the JLA PSP were pre-sorted into topics that were considered either within or beyond the scope of the exercise. For the analysis presented here, we used the entire dataset, regardless of whether the data were originally deemed in or out of scope. Combining the data from the JLA PSP with the
2019 workshops data, we undertook a thematic analysis ${ }^{25}$ Data were coded and mapped onto the JLA PSP top 10 priorities. Themes were conceptualised about people's experiences of living with MLTCs and the issues that were judged to be important. Data were managed and analysed using NVivo V.11. ${ }^{26}$

\section{Ethical considerations}

Ethical approval was not considered necessary for the JLA PSP exercise, PPI workshops or the review of JLA priorities. ${ }^{21}$

\section{Review of existing research priorities}

We undertook a scoping review of published JLA PSPs, to identify PSPs that related to older populations $(80+$ years) with MLTCs, and which were published since 2017. This updates a scoping exercise of JLA PSPs undertaken in 2017 when the JLA PSP on Multiple Conditions in Later Life was published. We also searched for ongoing research funded by the National Institute of Health Research (NIHR) and the UK Research Councils (UKRC).

\section{Searches}

We searched for all completed PSPs on the JLA website. For each completed PSP, the website and documentation (eg, reports, survey) were accessed to identify the questions published in both their 'shortlist' and 'longlist' of research priorities. We also searched for ongoing primary research projects funded by NIHR https://www.journalslibrary.nihr.ac.uk/programmes/ and the UK Research Council (https://gtr.ukri.org/) using the combinations of terms below:

1. 'older people'+'multi-morbidity'

2. 'older people'+ 'multiple conditions'

3. 'older populations'+ 'multi-morbidity'

4. 'older populations'+ 'multiple conditions'

\section{Screening, data extraction and synthesis}

All questions identified from completed JLA PSP shortlists and longlists, and all project titles of ongoing NIHR and UKRC projects, were extracted onto an excel spreadsheet. All questions and ongoing project titles were screened by one researcher to identify those that related to (a) older populations, and (b) older populations with more than one condition. Some PSPs had not published their longlist questions, and some only published all questions submitted to the initial survey. Therefore, it was not possible to screen the long list questions for every PSP. JLA PSP questions and ongoing NIHR/UKRC projects that related to older populations with multiple conditions were tabulated and summarised.

\section{RESULTS}

The JLA PSP published their priorities in 2019 (box 1). ${ }^{21}$ A full breakdown of the numbers of participants involved at each stage of the PSP exercise is reported elsewhere. ${ }^{21}$ Data analysed here originate from 115 people who contributed to the JLA PSP exercise, and 15 people who participated in the VOICE facilitated workshops.

\section{Qualitative data from the JLA PSP on multiple conditions in later life and PPI workshops}

Ten themes were identified (table 1).

\section{Organisation of health and social care services}

Older people and carers voiced their frustrations at the lack of joined-up care and support. Participants spoke of poor 
Table 1 Themes and supporting data

\begin{tabular}{|c|c|}
\hline Theme & Illustrative supporting quote(s) \\
\hline $\begin{array}{l}\text { Organisation of } \\
\text { health and social } \\
\text { care }\end{array}$ & $\begin{array}{l}\text { It's always, tell me your medical history or what conditions do you have at the start of an appointment. When you have to tell them about all } 6 \text {, there is no } \\
\text { time to speak to them about what you came in for, as you've only got } 10 \text { min! } \\
\text { Older person living with MLTC, Workshop } \\
\text { Need joined-up health and social care as they are so linked. In my stepmother's case, she needs one person to take responsibility for her care and coordinate } \\
\text { with the rest of the system and community. Friend/family member of older person living with MLTC, ID } 105\end{array}$ \\
\hline $\begin{array}{l}\text { Chal } \\
\text { prim }\end{array}$ & $\begin{array}{l}\text { I feel that there is nobody who sees them as a whole person, rather than someone with a single condition. Their care is all about their individual ailments, } \\
\text { not their overall health ... several health appointments in a single week to get warfarin level checked, blood pressure checked, arthritis clinic, hearing clinic, } \\
\text { rheumatology clinic, influenza jab. Each individual health professional is good, but they don't provide holistic care. How can I help my parents to manage this } \\
\text { better? } \\
\text { Friend/family member of older person living with MLTC, ID } 28\end{array}$ \\
\hline $\begin{array}{l}\text { ng } \\
\text { ons, pain } \\
\text { dication }\end{array}$ & $\begin{array}{l}\text { I would like to know a bit more about how things go together or if they don't. I mean when I had my heart attack, I must have been likely to have problems } \\
\text { long term and because I smoked and that. I think it would be helpful for people like me to know ... but I get its difficult and everyone is doing the best they } \\
\text { can. } \\
\text { Older person living with MLTC, Workshop } \\
\text { The medication I am given is ok, but it is impossible for me to understand or pronounce the even common tablets. ... I would like an easier way to refer to } \\
\text { these items? } \\
\text { Older person and carer, ID } 157\end{array}$ \\
\hline Carers' needs & $\begin{array}{l}\text { I think it is very easy to forget about the effect that looking after an older person with health problems can have on carers. This in return can eventually } \\
\text { impact on the effectiveness of the care being given. } \\
\text { Former carer of older person living with MLTC, ID } 76 \\
\text { My mam is the most complex and difficult person, she has delirium, and it got to the stage where she was calling me through the night. She wouldn't let } \\
\text { them (respite care) in. Multiple strokes, blindness and deafness, it was intense. I've lost everything. I feel invisible. I've done nothing but care. } \\
\text { Carer of older person living with MLTC, Workshop }\end{array}$ \\
\hline $\begin{array}{l}\text { Social isolation } \\
\text { mental health }\end{array}$ & $\begin{array}{l}\text { The year before the decline she was bowling } 5 \text { days a week and going out with friends twice a week. The lack of physical activity and social interaction has } \\
\text { had a noticeable impact on her physical and mental health. } \\
\text { Friend/family member of older person living with MLTC, ID } 77\end{array}$ \\
\hline $\begin{array}{l}\text { Financial impact } \\
\text { and funding for } \\
\text { care }\end{array}$ & $\begin{array}{l}\text { Yes, the financial costs of care is a constant worry. } \\
\text { Older person } 80+\text { living with MLTC, ID } 161 \\
\text { I think it's a problem that things like your teeth, feet, hearing and eyes and that have all gone private and none us can afford it so we don't go. } \\
\text { Older person living with MLTC, Workshop }\end{array}$ \\
\hline $\begin{array}{l}\text { Exercise and } \\
\text { physical activity }\end{array}$ & $\begin{array}{l}\text { It is fashionable to tell everyone to exercise-to walk and cycle but what about disabled people who are disadvantaged and need to be considered? } \\
\text { Older person } 80+\text { living with MLTC, ID } 165 \\
\text { I find it frustrating that health professionals seem to completely ignore this, the benefits of good diet, exercise and other therapies just because they are } \\
\text { dealing with an elderly person whose motivation to change is poor. Why is there not more emphasis put on the whole picture, encouraging elderly people to } \\
\text { eat well, socialise etc. } \\
\text { Friend/family member of older person living with MLTC, ID } 99\end{array}$ \\
\hline $\begin{array}{l}\text { Support for } \\
\text { independent living }\end{array}$ & $\begin{array}{l}\text { I want to be independent. Will do my best to be so. I try to live my own life in spite of my problems people who really need care ask for the least. } \\
\text { Older person living with MLTC, ID } 163\end{array}$ \\
\hline $\begin{array}{l}\text { Preventing } \\
\text { multimorbidity }\end{array}$ & $\begin{array}{l}\text { In some ways, we need to concentrate on the future. So if we concentrate on preventing multiple conditions that may help services for future generations. } \\
\text { Older person living with MLTC, Workshop }\end{array}$ \\
\hline
\end{tabular}

MLTC, multiple long-term condition.

communication between primary and secondary health services; between health and social care and between health/care services and local organisations (non-statutory) providing services (housing, home care, care homes). The consequence was relating their story multiple times, starting from the beginning at each appointment. This was attributed by some, to the absence of shared records.

There was a recognition that care and support needs are broader than health alone, and that housing and other carer support also need to be addressed. Discussions about housing or finances, for example, could be helpful. A keyworker, or care co-ordinator, could help them to navigate the system and ensure that their care is joined-up.

\section{Challenges in primary care}

Limited time available within standard primary care consultations did not accommodate the complexity of living with MLTCs. Participants felt that it was impossible for general practitioners (GPs) to provide holistic, person-centred care for someone with multiple conditions within a 10 minute appointment slot. Participants also reported a lack of understanding within primary care, of patient and carer needs and abilities, and limited appreciation of their lived experience of multiple conditions.

\section{Managing conditions, pain and medication}

Older people living with MLTCs expressed a wish to have a better understanding of their symptoms and prescribed medications, and of the interactions between their different health conditions. They also reported a need for more information to support treatment decisions and in particular, on alternatives to pharmaceutical treatments. The side-effects of multiple medication was, for some, a greater concern than the condition being treated. Pharmacists were proposed as a potential solution, to provide medication reviews.

\section{Carers' needs}

The burden placed on people supporting and caring for older people with multiple conditions was a major concern. Navigating the health and social care system to coordinate complex care services was perceived to be difficult and time-consuming. Carers wanted information about care and support services that was easy to access. In particular, they expressed a need for 
information on where to get help, and on their eligibility for time off work for caring.

Carers felt that it was important for them to be able to access information about the health conditions of the cared-for person, and the relationships between conditions, medications, interactions and side-effects. Distinguishing between symptoms of multiple conditions and side-effects of medication was a challenge for carers with no healthcare experience. Participants commented that simple guidelines about how to care for a person with multiple conditions would be valuable to carers.

\section{Social isolation and mental health}

Older people with multiple conditions talked about their experiences of becoming socially isolated, because of their increased needs and difficulties in getting out of the house. Participants talked about their experience of isolation, or their fear of it, together with its relationship with depression and overall adverse impact on mental and physical health. Some carers talked about the need for information and guidance on how to 'keep their spirits up' and how to support older people who were grieving, as well as expressing their own loss and isolation.

\section{Financial impact and funding for care}

Many participants were concerned about the costs of care that they face, worrying about having to sell their home, or find ways of financing the care needed. Participants who were still in work were concerned about taking time out of work to attend medical appointments and the possibility that they might lose their job and financial security.

\section{Exercise and physical activity for multiple conditions}

Many older people and carers expressed uncertainty about the type and amount of physical activity to undertake. Some thought that healthcare professionals did little to promote the importance of diet, exercise and staying active. Tailored advice from health professionals seemed to be lacking, particularly for the oldest old. For some, the problem of fatigue and lethargy, whether caused by the conditions or the medication taken, made it difficult to engage in physical activity and exercise. Those who were 'bed-bound' had found their symptoms and conditions worsen through inactivity.

\section{Support for independent living}

A strong desire to maintain independence was evident, but it was acknowledged that this was challenging in the presence of multiple conditions. Barriers to independent living included a paucity of (well-trained) staff to support independence, and the lack of timely access to equipment such as wheelchairs.

\section{Preventing multiple conditions}

Prevention was identified as a priority in the JLA PSP exercise on multiple conditions in later life. At the workshops held in July 2019 , older people and carers felt that this should be the number one priority.

\section{Review of existing research priorities}

Our review of JLA PSPs identified no new research priorities or ongoing projects specific to people with MLTCs aged over 80 years.

\section{DISCUSSION}

Our exploration of the views and experiences of older people who are living with MLTCs, and their carers, identified a number of key concerns. These concerns relate to access to care and support for both the patient and their carer, physical and mental health and well-being, and identifying opportunities for early prevention of multimorbidity. Most of these issues align with the research priorities identified by the JLA PSP. However, older people and carers identified three additional areas of importance to them, but which did not feature explicitly in the JLA top 10 priorities. These included the limitations of primary care, the financial costs of social care and understanding and managing their conditions at home (including multiple medications). Conversely, the JLA priorities relating to comprehensive geriatric assessment and falls risk were not mentioned by older people, nor carers. No data originating from older people contained any reference to frailty. Our review of JLA PSPs and ongoing funded studies identified no research projects specific to people with MLTCs aged over 80 years.

Concerns about access to timely, coordinated care for people with MLTCs are not novel. ${ }^{12} 13$ The importance of designing services around a person rather than their disease has been long recognised. ${ }^{1016}$ Yet the accounts of people in this study, and the research priorities identified in the PSP exercise, clearly indicate these challenges continue to be pertinent. This prompts the question of why so little progress has been made in addressing these issues and supporting older people with multiple conditions. A growing body of evidence clearly recommends what must change in practice to improve care for people living with MLTCs. This includes holistic and comprehensive assessment of needs, the use of coordinated case management, supporting self-management, multidisciplinary and interdisciplinary input into care, greater integration of clinical information between care settings, and training and support for practitioners. ${ }^{27-29}$ The ongoing concerns of people with MLTCs highlighted here signal that implementation of evidence into practice is slow. The continued organisation of medicine around single diseases is, most likely, a critical barrier. ${ }^{17}$ Greater integration of health and social care policy, specifically in relation to people with MLTCs, may also foster progress in practice. ${ }^{30}$

In addition to concerns about access to care, older people living with MLTCs highlighted the importance of several other issues. First, older people highlighted the significance of addressing social isolation. This aligns with recent evidence that social support is associated with better chances of survival for people with MLTCs. ${ }^{31}$ To date, however, the relationship between multimorbidity and social isolation is poorly understood. ${ }^{32}$ Older people also identified the need for support for their mental health and well-being. People living with MLTCs are more likely to experience poor mental health outcomes, ${ }^{33}$ adding yet further complexity to their healthcare needs. Recent calls for greater integration of mental health services with primary care underline the importance of this issue. ${ }^{34}$ Facilitating access to physical activity was another key area identified. This fits with emerging evidence that physical activity may improve quality of life for people with MLTCs. ${ }^{35}$ Finally, older people called for greater focus on the prevention of multimorbidity; a goal that mirrors recent calls to make this a priority as populations age. ${ }^{36} 37$

Other areas of importance were identified, but which did not feature explicitly in the JLA top 10 research priorities: the limitations of primary care in supporting people with MLTCs; the financial barriers to accessing social care in later life; and understanding and managing conditions at home (including multiple medications). The absence of these concerns from the top 10 may partly be due to the original scope of the JLA PSP, which was to identify uncertainties about interventions for people with 
MLTCs. Our analysis included all data from the JLA exercise, including topics that were originally considered out-of-scope. To some extent, these concerns about primary care and social care are encompassed within the broader top 10 priorities, particularly priorities 1 (optimising health, social care and voluntary sectors to meet needs) and 5 (enabling independent living effectively and acceptably). Other questions about maintaining independence, and medication adherence, also featured in the top 21 short list of priorities. Concerns relating to financing care and the lack of flexibility within primary care consultations reflect broader policy-level issues around access to care, but nonetheless signal key areas of practice and policy development for people with MLTCs. Future research may also wish to explore, in depth, the impact and consequences of these access barriers for people with MLTCs.

\section{Implications for future research and policy}

Our review of the JLA PSP research priorities and ongoing research identified no new priorities or projects specific to populations aged $80+$ with MLTCs. We identified a JLA PSP on frailty, as well as a UKRC project about sarcopenia and frailty that included a sub-sample of people aged $80+$ : both are likely to include people with MLTCs. However, the absence of any projects specifically about people aged $80+$ with MLTCs suggests there is scope for greater research focus on this population. A focus specifically on the lived experiences of people with MLTCs to inform service re-design may be an effective lever to foster implementation of evidence into practice. ${ }^{30}$ This may include greater understanding of how barriers in access to care (including primary and social care) impact people with MLTCs.

Concerns around both the limitations of primary care and social care costs warrant critical scrutiny on national policies by policy stakeholders. Standard 10 minute GP appointments have long been considered problematic ${ }^{38}$; for people with MLTCs, this practice may be especially detrimental to care. Social care reform, currently a priority for the UK government, must also pay close attention to the complex care needs of populations with MLTC.

\section{Strengths and limitations}

We scrutinised readily available data to further understand the experiences of people living with MLTCs. We did not have access to the original audio recordings or transcripts from the JLA PSP, thus limiting the scope and depth of our analysis. However, we were able to access the extracted data and upload this to NVivo V.11, identify key themes, and compare these to the top 10 research priorities identified by the JLA priority setting exercise. It is important to note that the JLA priorities were assessed against existing evidence. Any potential priorities that were already supported by evidence were excluded from the prioritisation list. This filter has not been applied to the data used here. Furthermore, we analysed all the data collected through the JLA PSP, including the data that were considered out-of-scope in the original priority setting exercise. As such, our findings provide a useful basis from which to identify future avenues of research that are aligned to the lived experiences of people living with MLTCs.

\section{CONCLUSION}

Older people living with MLTCs continue to experience care that is inadequate for their needs. A holistic approach will optimise quality of care and ensure wide-ranging needs-including physical, mental and social-are met. Healthcare must shift
Main messages

Older people with multiple long-term conditions experience care that is inadequate for their needs.

- This work identifies key concerns and priorities for this population.

- Future research priorities are recommended.

\section{Current research questions}

This work aimed to understand the lived experience of older people with multiple long-term conditions.

\section{What is already known on the subject}

- The number of people living with multiple long-term conditions is increasing globally.

- Health and care systems must adapt to the needs of this population.

- Understanding the experiences of older people with multiple long-term conditions is could inform service re-design and implementation.

from treating single conditions to supporting the whole person. Against a backdrop of rising multimorbidity, this is a critical message for practitioners across health and care settings. We also recommend key areas that should be given greater focus in both future research and policy development to inform effective and meaningful forms of support.

Acknowledgements The authors would like to acknowledge Stephen Hall, who contributed to the work of the James Lind Alliance Priority Setting Exercise on Multiple Long-Term Conditions. The views expressed are those of the authors and not necessarily those of the NIHR nor the Department of Health and Social Care.

Contributors GS undertook the review and co-wrote the paper; EB undertook the qualitative analysis and co-wrote the paper; $\mathrm{LC}$ undertook data collection for the workshops; DC designed and supervised the review and co-wrote the paper; CT cowrote the paper; $\mathrm{BH}$ undertook the qualitative analysis and co-wrote the paper. GS and $E B$ are the acting guarantors.

Funding This paper presents independent research funded by the National Institute for Health Research Policy Research Unit in Older People and Frailty (PR-PRU1217-21502)

Competing interests None declared.

Patient consent for publication Not applicable.

Provenance and peer review Not commissioned; externally peer reviewed.

Data availability statement All data relevant to the study are included in the article or uploaded as supplementary information.

Open access This is an open access article distributed in accordance with the Creative Commons Attribution 4.0 Unported (CC BY 4.0) license, which permits others to copy, redistribute, remix, transform and build upon this work for any purpose, provided the original work is properly cited, a link to the licence is given, and indication of whether changes were made. See: https://creativecommons.org/ licenses/by/4.0/.

\section{ORCID iD}

Gemma Spiers http://orcid.org/0000-0003-2121-4529

\section{REFERENCES}

1 Johnston MC, Crilly M, Black C, et al. Defining and measuring multimorbidity: a systematic review of systematic reviews. Eur J Public Health 2019;29:182-9. 
2 Garin N, Koyanagi A, Chatterji S, et al. Global multimorbidity patterns: a crosssectional, population-based, Multi-Country study. J Gerontol A Biol Sci Med Sci 2016;71:205-14.

3 Nguyen H, Manolova G, Daskalopoulou C, et al. Prevalence of multimorbidity in community settings: a systematic review and meta-analysis of observational studies. $J$ Comorb 2019:9:2235042X1987093.

4 Marengoni A, Angleman S, Melis R, et al. Aging with multimorbidity: a systematic review of the literature. Ageing Res Rev 2011;10:430-9.

5 Pathirana $\mathrm{TI}$, Jackson CA. Socioeconomic status and multimorbidity: a systematic review and meta-analysis. Aust N Z J Public Health 2018;42:186-94.

6 Ingram E, Ledden S, Beardon S, et al. Household and area-level social determinants of multimorbidity: a systematic review. J Epidemio/ Community Health 2021;75:232-41.

7 Makovski TT, Schmitz S, Zeegers MP, et al. Multimorbidity and quality of life: systematic literature review and meta-analysis. Ageing Res Rev 2019;53:100903.

8 Aubert CE, Schnipper JL, Fankhauser N, et al. Association of patterns of multimorbidity with length of stay: a multinational observational study. Medicine 2020:99:e21650.

9 Nunes BP, Flores TR, Mielke Gl, et al. Multimorbidity and mortality in older adults: a systematic review and meta-analysis. Arch Gerontol Geriatr 2016:67:130-8.

10 Whitty CJM, MacEwen C, Goddard A, et al. Rising to the challenge of multimorbidity. BMJ 2020;368:16964.

11 Damarell RA, Morgan DD, Tieman JJ. General practitioner strategies for managing patients with multimorbidity: a systematic review and thematic synthesis of qualitative research. BMC Fam Pract 2020;21:131.

12 van der Aa MJ, van den Broeke JR, Stronks K, et al. Patients with multimorbidity and their experiences with the healthcare process: a scoping review. J Comorb 2017;7:11-21.

13 Duguay C, Gallagher F, Fortin M. The experience of adults with multimorbidity: a qualitative study. J Comorb 2014;4:11-21.

14 Rosbach M, Andersen JS. Patient-experienced burden of treatment in patients with multimorbidity - A systematic review of qualitative data. PLoS One 2017; 12:e0179916.

15 Nissen NK, Aarhus R, Ørtenblad L. Dynamics of a specialized and complex health care system: Exploring general practitioners' management of multimorbidity. Chronic IIIn 2020:21:174239532092840

16 Monaco A, Palmer K, Marengoni A, et al. Integrated care for the management of ageing-related non-communicable diseases: current gaps and future directions. Aging Clin Exp Res 2020;32:1353-8.

17 Rijken M, Hujala A, van Ginneken E, et al. Managing multimorbidity: profiles of integrated care approaches targeting people with multiple chronic conditions in Europe. Health Policy 2018;122:44-52.

18 Griffith LE, Gruneir A, Fisher K, et al. Insights on multimorbidity and associated health service use and costs from three population-based studies of older adults in Ontario with diabetes, dementia and stroke. BMC Health Serv Res 2019;19:313.

19 Bähler C, Huber CA, Brüngger B, et al. Multimorbidity, health care utilization and costs in an elderly community-dwelling population: a claims data based observational study. BMC Health Serv Res 2015;15:23.
20 Soley-Bori M, Ashworth M, Bisquera A, et al. Impact of multimorbidity on healthcare costs and utilisation: a systematic review of the UK literature. Br J Gen Pract 2021;71:e39-e46.

21 Parker SG, Corner L, Laing K, et al. Priorities for research in multiple conditions in late life (multi-morbidity): findings from a James Lind alliance priority setting partnership. Age Ageing 2019;48:401-6.

22 Ploeg J, Matthew-Maich N, Fraser K, et al. Managing multiple chronic conditions in the community: a Canadian qualitative study of the experiences of older adults, family caregivers and healthcare providers. BMC Geriatr 2017;17:40.

23 Porter T, Ong BN, Sanders T. Living with multimorbidity? the lived experience of multiple chronic conditions in later life. Health 2020;24:701-18.

24 Hasardzhiev S, Mendão L, Nolte W, et al. Managing multimorbidity: how can the patient experience be improved? J Comorb 2016;6:28-32.

25 Ritchie J, Lewis J. Qualitative research practice: a guide for social science students and researchers. SAGE, 2003.

26 QSR International Pty Ltd. NVivo11, released 2015, 2015. Available: https://www. qsrinternational.com/nvivo-qualitative-data-analysis-software/home

27 Poitras M-E, Maltais M-E, Bestard-Denommé L, et al. What are the effective elements in patient-centered and multimorbidity care? A scoping review. BMC Health Serv Res 2018;18:446.

28 Palmer K, Marengoni A, Forjaz MJ, et al. Multimorbidity care model: recommendations from the consensus meeting of the joint action on chronic diseases and promoting healthy ageing across the life cycle (JA-CHRODIS). Health Policy 2018;122:4-11.

29 Boyd CM, Fortin M. Future of multimorbidity research: how should understanding of multimorbidity inform health system design? Public Health Rev 2010;32:451-74.

30 Pearson-Stuttard J, Ezzati M, Gregg EW. Multimorbidity-a defining challenge for health systems. Lancet Public Health 2019;4:e599-600.

31 Olaya $B$, Domènech-Abella J, Moneta MV, et al. All-Cause mortality and multimorbidity in older adults: the role of social support and loneliness. Exp Gerontol 2017;99:120-6.

32 Hajek A, Kretzler B, König H-H. Multimorbidity, loneliness, and social isolation. A systematic review. Int J Environ Res Public Health 2020;17. doi:10.3390/ ijerph17228688. [Epub ahead of print: 2311 2020].

33 Arokiasamy $\mathrm{P}$, Uttamacharya $U$, Jain $\mathrm{K}$, et al. The impact of multimorbidity on adult physical and mental health in low- and middle-income countries: what does the study on global ageing and adult health (SAGE) reveal? BMC Med 2015;13:178. doi:10.1186/s12916-015-0402-8

34 Langan J, Mercer SW, Smith DJ. Multimorbidity and mental health: can psychiatry rise to the challenge? Br J Psychiatry 2013:202:391-3.

35 Subramaniam M, Zhang Y, Lau JH, et al. Patterns of physical activity and healthrelated quality of life amongst patients with multimorbidity in a multi-ethnic Asian population. BMC Public Health 2019;19:1612

36 Navickas R, Petric V-K, Feigl AB, et al. Multimorbidity: what do we know? what should we do? J Comorb 2016:6:4-11.

37 Head A, Fleming K, Kypridemos C, et al. Multimorbidity: the case for prevention. $J$ Epidemiol Community Health 2021;75:242-4.

38 Flaxman P. The 10-minute appointment. Br J Gen Pract 2015;65:573.3-4. 